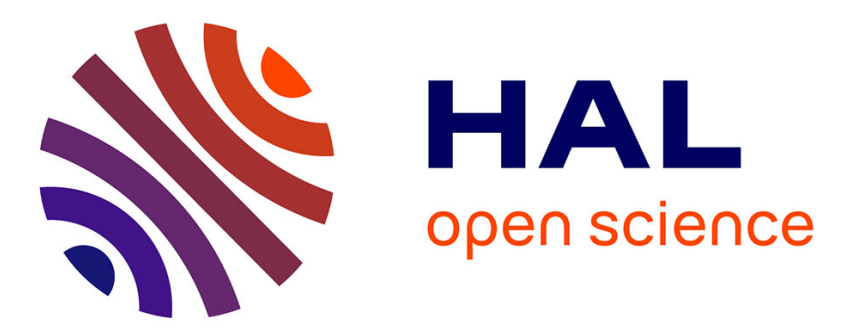

\title{
Tailor-made surface plasmon polaritons above the bulk plasma frequency: a design strategy for indium tin oxide S Brand, R A Abram, M A Kaliteevski
}

\section{To cite this version:}

S Brand, R A Abram, M A Kaliteevski. Tailor-made surface plasmon polaritons above the bulk plasma frequency: a design strategy for indium tin oxide. Journal of Physics D: Applied Physics, 2010, 43 (14), pp.145104. 10.1088/0022-3727/43/14/145104 . hal-00569571

\section{HAL Id: hal-00569571 \\ https://hal.science/hal-00569571}

Submitted on 25 Feb 2011

HAL is a multi-disciplinary open access archive for the deposit and dissemination of scientific research documents, whether they are published or not. The documents may come from teaching and research institutions in France or abroad, or from public or private research centers.
L'archive ouverte pluridisciplinaire HAL, est destinée au dépôt et à la diffusion de documents scientifiques de niveau recherche, publiés ou non, émanant des établissements d'enseignement et de recherche français ou étrangers, des laboratoires publics ou privés. 
Tailor-made surface plasmon polaritons above the bulk plasma frequency: a design strategy for indium tin oxide

S Brand, R A Abram and M A Kaliteevski

Department of Physics, Durham University, South Road, Durham DH1 3LE, UK.

\begin{abstract}
A simple phase-matching approach is employed as a design aid to engineer surface plasmon polariton states at the interface of an indium tin oxide layer on the top of a Bragg reflector. By altering the details of the reflector, and in particular the ordering of the layers and the thickness of the layer adjacent to the indium tin oxide, it is possible to readily adjust the energy of these states. Examples of structures engineered to give rise to distinctive features in the reflectivity spectra above the bulk screened plasma frequency for states of both possible polarizations are presented.
\end{abstract}

Short title: Surface polaritons above the bulk plasma frequency in ITO

PACS numbers: 41.20 Jb, 42.70 Qs, 73.20 Mf, $78.68+\mathrm{m}$ 


\section{Introduction}

Surface plasmon-related excitations, which may have advantageous or deleterious consequences, depending on circumstances, have most often been studied at interfaces involving silver or gold but Rhodes et al have experimentally demonstrated the presence of both bulk and surface plasmon polaritons (SPPs) in indium tin oxide (ITO) on a glass substrate [1,2]. ITO is currently particularly useful as an electrode in a variety of optoelectronic devices and other oxides, such as $\mathrm{ZnO}$, may become important for such applications. The existence of SPPs in conducting oxide films was confirmed by Michelotti et al [3] in the case of ITO, although they were unable to detect their signature in an alternative series of conducting oxide $\mathrm{ZnO}: \mathrm{Al}$ films, and additional results on ITO have more recently been reported by Losego et al [4]. All of these reflectivity experiments were carried out employing a standard prism-coupling technique in order to access the SPP states using TM-polarized light in which the electric field is polarized in the plane of incidence. There are no associated SPPs at the interface of these simple conductor/substrate structures with the orthogonal TE polarization in which the electric field is polarized parallel to the interface as these are precluded by the boundary conditions. However, we have recently demonstrated theoretically that both TM- and TE-polarized optical Tamm states or SPPs should be observable above the plasma frequency $\left(\omega_{p}\right)$ in a system in which the conducting film is on the surface of a Bragg relector, and further, that such states can be revealed without the use of prism coupling [5]. In that work, however, we considered only an idealised conductor with a frequency-dependent permittivity of the form $\varepsilon(\omega)=1-\omega_{p}^{2} / \omega\left(\omega+i \omega_{c}\right)$, where $\omega_{c}$ is the loss-associated collision frequency term. In this work we consider a system employing a permittivity consistent with the experimental work on ITO of Rhodes et al [2] and Michelotti et al [3], and give a more detailed analysis of the conditions under which the SPPs are observable. Brief and more extended reviews of SPPs can be found in the works of Barnes et al [6] and Pitarke et al [7] respectively. 


\section{Background considerations}

In previous work Kaliteevski et al [8] showed that in order to obtain an eigenmode or surface state at an interface between a metal (on the left) and Bragg reflector (on the right) it is necessary to fulfil the condition that $r_{\text {left }} r_{\text {right }}=1$ where $r_{\text {left }}, r_{\text {right }}$ represent electric field amplitude reflection coefficients for light travelling from right to left, and left to right respectively at a virtual interface within the Bragg reflector considered to be an infinitesimal distance from the metal interface. In the simplest cases this means that $r=r_{\text {left }}=r_{\text {right }}= \pm 1$. The specific case where $r \approx-1$ was considered in that work, corresponding to the situation in which the phase of the complex reflection coefficient is approximately $\pi$ which occurs when considering reflection from a metal at an energy well below the plasma frequency and near the centre of the photonic band gap (PBG) of a Bragg reflector with a high refractive index layer adjacent to the metal. In this case the existence of the PBG ensures that states at energies within the PBG are confined by the Bragg reflector on one side of the interface and the evanescent nature of the state below the plasma frequency in the metal does so on the other. Subsequent experiments demonstrated the existence of such states at the interface of a gold/GaAs-AlGaAs Bragg reflector structure [9]. The alternative situation in which $r \approx+1$, and hence the phase change is approximately zero for both reflections was considered in our more recent work [5] dealing with surface states above the plasma frequency but within the PBG of the Bragg reflector: in that case the layer adjacent to the metal had the lower refractive index (leading to $\left.r_{\text {right }}=+1\right)$ and total internal reflection at the interface with the metal is responsible for the existence of the state. Similar arguments were utilised earlier by Kavokin et al [10] who considered lossless modes at the interface between two different periodic dielectric structures. More generally, it follows that the requirement for a surface state can be stated as

$$
\phi_{\text {left }}+\phi_{\text {right }}=0 \quad(+ \text { integer } \times 2 \pi)
$$

where $\phi_{\text {left }}$ and $\phi_{\text {right }}$ are the respective phases of the two complex amplitude reflection coefficients and this leads to a complete family of more general phase matching situations in 
which surface states may be found. Although eqn. (1) does not give any information concerning the detailed form of the resultant surface state it represents a very simple and useful criterion as an aid to the design of structures exhibiting such states.

In what follows we consider the case of an ITO layer on a Bragg reflector, as illustrated in figure 1, and show that such structures can be designed to exhibit SPP features above the (screened) ITO plasma frequency in both TE and TM polarisations. For ITO we take the permittivity to be essentially of the form given by Rhodes et al [2] :

$$
\varepsilon(\omega)=\varepsilon_{\infty}\left(1-\frac{\omega_{p}^{2}}{\omega\left(\omega+i \omega_{c}\right)}\right)
$$

where $\varepsilon_{\infty}=3.8, \hbar \omega_{p}=2.19 / \sqrt{\varepsilon_{\infty}} \equiv 1.123 \mathrm{eV}$ and $\hbar \omega_{c}=0.111 \mathrm{eV}$. These workers were able to obtain good agreement between experiment and calculated reflectivity coefficients with these parameters, which are similar to those reported by Michelotti et al [3]

$\left(\varepsilon_{\infty}=3.9, \hbar \omega_{p}=1.909 / \sqrt{\varepsilon_{\infty}}=0.967 \mathrm{eV}, \hbar \omega_{c}=0.118 \mathrm{eV}\right)$. For the purposes of the current calculations a Bragg reflector consisting of $\mathrm{SiO}_{2}$ and $\mathrm{TiO}_{2}$ layers is employed. In general the refractive index of these materials is complex and depends upon frequency (see e.g. Lee and Yao [11]) but in the infra-red regime considered in the present work we take that of $\mathrm{SiO}_{2}$ to be 1.47 and use a value of 2.37 for $\mathrm{TiO}_{2}$.

We note that as well as the experimental justification supplied by Sasin el al [9] in the near infra red region, the additional results of Guo et al [12], who considered the GHz response of microstrip transmission lines, confirms the general utility of the phase-matching approach for the design and manipulation of optical Tamm states.

\section{Phase calculations}

The phase associated with reflection from the ITO layer, $\phi_{\text {left }}$, depends on the thickness of the ITO layer, the refractive index of the top layer in the Bragg stack, the angle of incidence and the energy and polarization of light considered. In the case of a finite thickness ITO layer it 
also depends on the refractive index in the region following the ITO, which is taken to be air. As an illustration of the value of $\phi_{\text {left }}$, associated with reflection from the ITO, we show in figures $2 \mathrm{a}$ ), b) results for both TM and TE polarizations as a function of energy at a particular fixed angle. For these calculations a high index $\mathrm{TiO}_{2}$ layer is at the top of the Bragg reflector: results for $\phi_{\text {left }}$ with the lower index material at the interface with ITO are different in detail but of the same form. As the phase depends on the thickness of the ITO layer we show results calculated via the standard Fresnel equations for an infinite system as well as for a finite structure with an arbitrary $200 \mathrm{~nm}$ layer of ITO followed by air. In the latter case the phase is evaluated using a transfer matrix calculation. Results are shown for both a lossless $\left(\omega_{c}=0\right)$ representation of the ITO permittivity as well as with the full equation (2) loss term included. In the case of TM polarization the standard Fresnel equation calculation without losses for the ITO (curve FNL in figure 2 (a)) shows predictable behaviour. At high energy, corresponding to light at high frequency and consequently in a regime with relatively high refractive index (albeit with $n<1$ ), the set angle of $20^{\circ}$ is below both the critical angle, $\theta_{C}$, and the Brewster angle, $\theta_{B}$, and the phase $\phi_{\text {left }}=0$. However, when the energy/frequency is reduced far enough, $\varepsilon(\omega)$ is such that the refractive index is reduced to the point where $\theta_{B}=20^{\circ}$ and the phase jumps discontinuously to $180^{\circ}$ : this occurs at an energy $\approx 1.252 \mathrm{eV}$, when $n \approx 0.86$. At a slightly smaller energy $\approx 1.235 \mathrm{eV}$, when $n \approx 0.81$, the corresponding frequency of the light is such that the critical angle is reduced to $\theta_{C}=20^{\circ}$, the set angle, and the phase then drops rapidly below $180^{\circ}$ as the energy is reduced further, tending to the expected value of $0^{\circ}$ at an energy corresponding to the plasma frequency of $1.123 \mathrm{eV}$ and continuing to fall towards $-180^{\circ}$ far below this energy. It is in the regime of energy above the bulk plasma frequency but below the energy for which $\theta_{C}$ equals the angle of incidence that SPPs of the type we are considering can potentially be seen. In the simpler case of TE polarization with no losses, the Fresnel result is that $\phi_{\text {left }}=0$ until the energy corresponds to that for $\theta_{C}=20^{\circ}$ and the phase then gradually shifts 
down towards $-180^{\circ}$. The results are somewhat modified for the finite width ITO structure and with losses included but all results are very similar in the neighbourhood of and below that corresponding to the plasma frequency.

We now consider an illustration of results for $\phi_{\text {right }}$, the phase associated with reflection from the Bragg reflector. We take the reflector to be formed from $188 \mathrm{~nm}$ layers of $\mathrm{SiO}_{2}$ and $116 \mathrm{~nm}$ layers of $\mathrm{TiO}_{2}$ on a $\mathrm{SiO}_{2}$ substrate (refractive index $=1.47$ ). The structure is designed to have a photonic band gap centred at $1.123 \mathrm{eV}$ at normal incidence and this results in a photonic band gap between $\approx 0.95 \rightarrow 1.29 \mathrm{eV}$ (for a Bragg reflector of infinite extent). Figures $3 \mathrm{a}$ ), b) show $\phi_{\text {right }}$ as a function of energy for a structure consisting of 20 layer pairs on a $\mathrm{SiO}_{2}$ substrate as a function of the thickness, $d$, and composition of the final layer. The results are calculated using a standard transfer matrix approach. By altering the material of the top layer and also the thickness of this final layer it is possible to adjust $\phi_{\text {right }}$ to have any desired value in the range \pm $\pi$ at a chosen energy within the PBG. When the top layer in the reflector is composed of low refractive index material with $d=1.0 \times \lambda /\left(4 n_{1}\right)$ the phase at the centre of the $\mathrm{PBG}$ is $0^{\circ}$ and alternatively, when a quarter-wave layer of the high refractive index material is present the phase at the centre of the PBG is $\phi_{\text {right }}= \pm 180^{\circ}$ (which are equivalent values). We note that in the PBG region, when incoming light is totally reflected, the change in phase at a given frequency in response to a change in width is directly related to the change in optical path length due to the related round-trip distance. At normal incidence there is, of course, no distinction between the results for TE and TM polarization. At a more general angle of incidence the photonic band gap region shifts to higher energies and there is a difference in the detailed results for the two polarizations. Note that the centre of the PBG can be shifted relative to the plasma frequency of the ITO by altering the structure of the Bragg reflector. Thus there is considerable flexibility with regard to the adjustment of the phase $\phi_{\text {right }}$ associated with the Bragg reflector at any particular energy through the choice of the PBG or angle of incidence or 
polarization. This flexibility can be employed to tailor-make a structure to satisfy equation (1) at a chosen energy for a particular metallic surface layer and given polarization.

The general form of the results in figure $2 a$ ), b) indicate that in order to obtain a SPP well-confined by internal reflection at the ITO/Bragg reflector interface, the phase $\phi_{\text {left }}$ will be relatively small in magnitude, irrespective of polarization. If the loss term is neglected then $\phi_{\text {left }}$ can be large, approaching $\pi$ when the angle of incidence $\left(20^{\circ}\right.$ here $)$ is just above the critical angle in the case of TM polarization, but in such a case confinement will be poor, so angles of incidence significantly larger than $\theta_{C}$ despite the smaller $\phi_{\text {left }}$ are to be preferred. When losses are included $\phi_{\text {left }}$ is always relatively small near, but above, the plasma frequency, having a negative value in the case of TE and a positive value in the case of TM polarization. Thus it is clear that the situation in which the high index, quarter-wave material of the Bragg reflector is at the interface provides an unfavourable scenario for the formation of SPPs near the plasma frequency if the band gap is centred at the plasma frequency: Figure 3a) shows that the phase $\phi_{\text {right }} \approx \pm 180^{\circ}$ in this region and with $\phi_{\text {left }}$ small, equation (1) cannot be satisfied. On the other hand, figure $3 \mathrm{~b}$ ) demonstrates that $\phi_{\text {right }}$ is relatively small close to the centre of the gap and hence equation (1) can be satisfied at some energy near $\hbar \omega_{p}$, which explains the choice of low index material at the interface in our earlier work [5], although the detailed considerations were slightly different due to the simpler form of $\varepsilon(\omega)$ in that report.

\section{4 (a). Results: TM SPPs above the plasma frequency}

Despite the arguments made in Section 3, it is possible to produce structures that support SPPs in which the Bragg reflector is such that the top layer is formed from the high index $\mathrm{TiO}_{2}$ material. In fact there is some advantage in choosing to position the high index material at the interface with ITO because that reduces the critical angle at that interface and enhances the confinement of the SPP. The general form of $\phi_{\text {right }}$ as shown in figure 3 a) does 
produce an unfavourable arrangement for the satisfaction of equation (1) if that particular reflector is employed but an appropriate phase shift can be produced if the thickness of the top layer is increased, a reflector with a PBG centred at a lower energy is employed or some combination of both adjustments is made. To illustrate this point we choose to utilise a reflector designed to have a PBG of $1 \mathrm{eV}$ and maintain a quarter-wave $\mathrm{TiO}_{2}$ layer bordering the ITO. In this case the respective thicknesses of the $\mathrm{TiO}_{2}$ and $\mathrm{SiO}_{2}$ layers are $131 \mathrm{~nm}$ and $211 \mathrm{~nm}$ and the normal incidence $\mathrm{PBG}$ is from $\approx 0.85 \mathrm{eV} \rightarrow 1.15 \mathrm{eV}$, largely below $\hbar \omega_{p} \equiv 1.123 \mathrm{eV}$. However, at high angles of incidence the PBG is effectively shifted to higher energies, the plasmon energy falls further within the PBG and equation (1) can more easily be satisfied above the plasma frequency. This is illustrated in figure 4 which shows a sharp feature in the (power) reflection coefficient associated with the SPP at $1.230 \mathrm{eV}$. In order to demonstrate the existence of this state clearly, the collision term has been artificially reduced to $0.01 \omega_{c}$, the incident angle at the external air/ITO interface, $\theta_{e}$, has been chosen as $54.3^{\circ}$ (this external air/ITO interface angle corresponds to the internal $\mathrm{TiO}_{2}$ angle $\theta_{i}=20^{\circ}$ employed in constructing figure 2) and a relatively thick, $w=1650 \mathrm{~nm}$ ITO layer is used. Under these circumstances the SPP energy effectively corresponds to the position of the narrow minimum and we can compare this with the predicted energy of the SPP found from equation (1) (note that $\phi_{\text {left }}$ and $\phi_{\text {right }}$ are evaluated at the internal angle, $\theta_{i}$, within the $\mathrm{TiO}_{2}$ ). The phase-matching approach and also the semiinfinite ITO/Bragg reflector structure result obtained using the complex photonic bandstructure method described in Brand et al [5] both predict the energy of the SPP to be $1.229 \mathrm{eV}$ when the loss term is neglected, in excellent agreement with the reflectivity result. Figure 4 also shows the reflection coefficient associated with the 40-layer Bragg reflector on the $\mathrm{SiO}_{2}$ substrate without the ITO cap layer to make it clear that the SPP feature lies just below the PBG edge. If the value of $d$ is increased then the SPP moves to lower energy and vice versa. The form of the 
variation in the SPP energy as a function of Bragg reflector top layer width was presented in Kaliteevski et al [8] without discussing the specific form of the individual phase variations.

In order to demonstrate the detailed form of the SPP we reduce the loss term to $0.001 \omega_{c}$, extend the thickness of the ITO to $w=2700 \mathrm{~nm}$ and also consider a $1000 \mathrm{~nm}$ thick region of the air to the left of the ITO. A plot of the resultant component of the electric field intensity $\left(E_{\tan }^{2}\right)$ tangential to the interface as calculated from the transfer matrix approach is shown in figure 5 . This clearly reveals the characteristic properties of a surface state localized at the ITO/Bragg reflector interface. We note that at the SPP energy, the value of the refractive index (with loss term neglected) of the ITO is 0.795 with a corresponding external critical angle at the air/ITO interface of $\theta_{e c}=52.6^{\circ}$, below that of the external angle $\theta_{e}=54.3^{\circ}$ considered. If the thickness of the ITO is increased we find that there is eventually insufficient penetration of the light through the ITO due to the evanescent nature of the electromagnetic field at the air/ITO interface, and it becomes more difficult to observe the feature due to the SPP at the ITO/Bragg reflector interface.

The previous results were chosen to understand the underlying nature of the SPP states more clearly but relate to an unrealistic situation with regard to the ITO loss term. Consequently we now consider a more realistic scenario in which the full loss term of equation (2) is included and the corresponding results are therefore representative of what would be expected in an actual experiment. This necessitates the use of much thinner ITO layers, otherwise there is insufficient penetration of light to access the state at the ITO/Bragg reflector interface. Figure 6 shows the calculated reflection coefficient at the same external angle in air as used previously, $\theta_{e}=54.3^{\circ}$, for a range of ITO layer thicknesses. The structure is otherwise as before. In the case of very thin layers an obvious feature is present very close to the centre of the PBG (the structure has been designed such that the feature will appear at the centre of the gap at the 
chosen angle) at an energy corresponding to that of the screened bulk plasmon. As $w$ is increased the dip in the reflection coefficient becomes more prominent, shifts to higher energy and we see a near zero reflection coefficient when the ITO layer width is $w=120 \mathrm{~nm}$. The dip continues to move towards the energy of the SPP as the thickness of the ITO layer is increased but the effects of the high loss term eventually dominate the reflectivity, the penetration of the light to the ITO/Bragg reflector interface is suppressed and the initial strong feature in the reflectivity vanishes. We note that when a large loss term is present and a much broader feature is obtained than in the previous idealised calculations, we no longer expect the minimum in the reflection coefficient to coincide with the SPP energy although it does approach this value as the ITO thickness is increased before finally disappearing. For comparison, results are also displayed for the situation with a Bragg reflector and no ITO over-layer and also for a bare 120 nm ITO layer on $\mathrm{SiO}_{2}$ substrate. We note that the shift in the reflection minimum away from the bulk plasmon value and towards that of the SPP as the ITO thickness is increased is similar to that demonstrated by Rhodes et al [2] except that in their case the shift was downwards towards a more conventional lower energy SPP and prism coupling was required in order to obtain the results.

\section{4 (b). Results: TE SPPs above the plasma frequency}

In the case of TE polarization the general form of figure $2 \mathrm{~b}$ ), indicates that the phase $\phi_{\text {left }}$ is negative above the critical angle at the ITO/dielectric interface irrespective of the details concerning ITO layer thickness, loss term and dielectric material. Also, the results of figure $3 \mathrm{~b}$ ) illustrate that the phase $\phi_{\text {right }}$ is always positive at energies above the PBG centre when a low index quarter-wave layer is present at the top of the Bragg reflector. Thus equation (1) should be easily satisfied if the low index material in the Bragg reflector is adjacent to the ITO. To examine this case in more detail we reverse the ordering of the final layer compared to the calculations in the previous section, but otherwise employ the same basic 20 layer-pair Bragg reflector with $\mathrm{PBG}$ centred on $1 \mathrm{eV}$. 
To begin, we first confirm that the expected localized TE SPP exists above the bulk screened plasmon energy, and for the purposes of our calculations we choose an external air/ITO interface angle of incidence of $\theta_{e}=50^{\circ}$ (this external air/ITO interface angle corresponds to an internal $\mathrm{SiO}_{2}$ angle $\theta_{i}=31.4^{\circ}$ ). The semi-infinite ITO/Bragg reflector calculation [5] gives the resultant SPP energy to be $1.202 \mathrm{eV}$, whereas the phase-matching approach indicates that the solution is to be expected at $1.203 \mathrm{eV}$. These results can be compared with the transfer matrix reflection coefficient plot of figure 7 which shows a sharp dip at an energy of $1.203 \mathrm{eV}$. In order to obtain definitive results we have employed an artificially reduced loss term of $0.01 \omega_{c}$ and an associated large ITO thickness of $1210 \mathrm{~nm}$, which ensures good localization of the SPP and a sharp feature in the reflectivity plot. The associated electric field intensity plot, calculated via the transfer matrix approach, is shown in figure 8 and this clearly demonstrates that the feature is due to an SPP localized at the ITO Bragg reflector interface. At the SPP energy the value of the refractive index (with loss term neglected) of the ITO is 0.698 with a corresponding external critical angle in the air at the air/ITO interface of $\theta_{e c}=44.3^{\circ}$, below that of the external angle considered, $\theta_{e}=50^{\circ}$. Thus it is again necessary to employ a carefully chosen balance of ITO layer thickness and loss term in order to reveal the nature of the SPP while avoiding a situation in which the evanescent field at the air/ITO interface is such that the SPP cannot be effectively accessed. Again, as expected, calculations confirm that the SPP moves to lower or higher energy when the value of $d$ is increased or decreased respectively. It can also be observed in figure 7 that there is a minor dip in the reflection plot at about $1.26 \mathrm{eV}$. The transfer matrix calculation of the associated $E$-field intensity at this energy reveals a peak at the centre of the ITO layer, as opposed to localization at the ITO/BR interface. The position of the feature shifts to higher energy as the ITO layer thickness is decreased and vice versa as the confinement of the associated state is altered. We have also computed results for a realistic situation with the full loss term included. Figure 9 illustrates the calculated reflection coefficient for TE polarization as a function of 
energy for a range of ITO layer thicknesses. The external angle of incidence is $50^{\circ}$. For this polarization the feature associated with the bulk plasmon at $1.123 \mathrm{eV}$ is weaker and less sharp than in the TM case for the smaller ITO layer thicknesses but a very distinctive reflectivity minimum associated with the presence of the SPP develops as the ITO thickness increases up to $260 \mathrm{~nm}$. The energy at this minimum is just under $1.2 \mathrm{eV}$, very close to the idealised value of $1.203 \mathrm{eV}$ obtained from the previous essentially loss-free calculations. We note that the feature becomes less prominent as the ITO layer becomes thicker and penetration to the ITO/Bragg reflector interface is reduced.

\section{Conclusions}

We have demonstrated that significant features are to be expected in the reflectivity spectra of indium tin oxide layers on a Bragg reflector as a consequence of surface plasmon polaritons at the interface. It is not necessary to employ a prism coupling technique, as is normally required, in order to observe the effects of the presence of these states although additional distinctive features (not described in this work) can be seen in that case. By an appropriate choice of structure, the design of which is aided by consideration of the simple phase matching condition $\phi_{\text {left }}+\phi_{\text {right }}=0$, the SPP energy can be easily adjusted and, in particular, can be positioned above the bulk screened plasmon energy. This is in contrast to the normal situation without the reflector where the SPP is found below the bulk plasmon energy. In addition, the position of the SPP can be readily shifted by an appropriate choice of ITO layer thickness and that of the material forming the dielectric layer at the top of the reflector. Although the particular conditions employed have been designed to enhance the distinctive nature of the reflectivity response presented, the general features described should be observable over a range of experimental conditions and the general design approach is applicable to other systems. 


\section{References}

[1] Rhodes C, Franzen S, Maria J-P, Losego M, Leonard D N, Laughlin B, Duscher G and Weibel S, 2006 J. Appl. Phys. 100054905

[2] Rhodes C, Cerruti M, Efremenko A, Losego M, Aspnes D E, Maria J-P and Franzen S, 2008 J. Appl. Phys. 103093108

[3] Michelotti F, Dominici L, Descrovi E, Danz N and Menchini F, 2009 Opt. Letts. 34 839-41

[4] Losego M D, Efremenko A Y, Rhodes C L, Cerruti M G, Franzen S and Maria J-P, 2009 J. Appl. Phys. 106024903

[5] Brand S, Kaliteevski M A and Abram R A, 2009 Phys. Rev. B79 085416

[6] Barnes W L, Dereux A and Ebbesen T W, 2003 Nature 424 824-30

[7] Pitarke J M, Silkin V M, Chulkov E V and Echenique P M, 2007 Rep. Prog. Phys. 70 1-87

[8] Kaliteevski M, Iorsh I, Brand S, Abram R A, Chamberlain J M, Kavokin A V and Shelykh I A, 2007 Phys. Rev. B76 165415

[9] Sasin M E, Seisyan R P, Kaliteevski M, Brand S, Abram R A, Chamberlain J M, Vasil'ev A P, Mikhrin V S and Kavokin A V, Appl. Phys. Letts. 92 251112(2008)

[10] Kavokin A V, Shelykh I A and Malpuech G, 2005 Phys. Rev. B72 233102

[11] Lee H-Yand Yao T, 2004 J. Kor. Phys. Soc. 44 387-92

[12] Guo J, Sun Y, Zhang Y, Li H, Jiang H and Chen H, 2008 Phys. Rev. E 78026607 


\section{Figure captions}

FIG. 1 The basic structure considered, consisting of an ITO layer of thickness $w$ on top of a multi-pair Bragg reflector on $\mathrm{SiO}_{2}$ substrate. The refractive indices of the Bragg reflector layers are $n_{1}$ and $n_{2}$ and the top layer in the stack has width $d$. The refractive index of the substrate is $n_{s}$. The external angle of incidence, $\theta_{e}$, for incoming light and the corresponding internal angle, $\theta_{i}$, within the first Bragg reflector layer are indicated. The vertical arrow indicates the interface at which the phase results in Figures 2 and 3 are calculated (see text).

FIG. 2 The phase, $\phi_{\text {left }}$, as a function of energy when light is incident on the ITO from a high index $\left(\mathrm{TiO}_{2}, n=2.37\right)$ layer at a fixed angle of $20^{\circ}$. (a) shows the results for TM polarization, and (b) is for TE polarization. F indicates an infinite structure Fresnel equation calculation with (L) and without (NL) losses $\left(\omega_{c}=0\right)$. Transfer matrix calculations for a finite $200 \mathrm{~nm}$ ITO layer with and without the loss term included are also shown. The points at which the refractive index of the ITO (which decreases from a value near $\sqrt{3.8}$ at very high energies towards 0 as the energy approaches $1.123 \mathrm{eV}$ from above if losses are neglected) is such that $\theta_{C}$ and $\theta_{B}$ become equal to $20^{\circ}$ are indicated by arrows. At high energies the fixed angle of $20^{\circ}$ is below both $\theta_{B}$ and $\theta_{C}$ and hence $\phi_{\text {left }}=0$ for both polarizations. The large arrows shown here and in the following figures indicate the general direction of incident light relative to the original structure shown in Figure 1.

FIG. 3 The phase, $\phi_{\text {right }}$, as a function of the energy of the incident light. Results are given for different thicknesses of the layer at the top of the Bragg reflector, $d=p \times \lambda /\left(4 n_{1}\right)$ and are labelled by the value of $p$. In (a) $n_{1}=2.37$ corresponds to that for the higher refractive index material $\mathrm{TiO}_{2}$ and (b) shows results for the situation in which $n_{1}=$ 
1.47, corresponding to that of the lower index material, $\mathrm{SiO}_{2}$. The results are for normal incidence and hence are independent of polarization. For an infinite Bragg reflector the PBG is from about $0.95 \rightarrow 1.29 \mathrm{eV}$, centred on $1.123 \mathrm{eV}$. In both cases the refractive index of the substrate is taken to be $n_{s}=1.47$.

FIG. 4 TM polarization. The reflection coefficient as a function of energy at an external air/interface angle of $54.3^{\circ}$. Results for the air/Bragg reflector/glass substrate (Bragg) and air/ITO(1650 nm)/Bragg reflector/glass substrate (BraggITO) structures are shown. A distinct feature associated with an SPP at the ITO/Bragg reflector interface is present at $1.230 \mathrm{eV}$. A nominal collision term of $0.01 \omega_{c}$ was employed in the calculations.

FIG. 5 A plot of the tangential component of the electric field intensity, $E_{\text {tan }}^{2}$ (relative), associated with the TM SPP state. The peak at $3.7 \mu \mathrm{m}$, is exactly at the interface between the ITO and the Bragg reflector. The field intensity is plotted relative to that of the incident wave.

FIG. 6 The TM reflection coefficient as a function of energy for $w=1 \mathrm{~nm}, 10 \mathrm{~nm}, 20 \mathrm{~nm}$, $50 \mathrm{~nm}, 120 \mathrm{~nm}$ and $400 \mathrm{~nm}$ thick ITO layers on a 40 layer $\mathrm{TiO}_{2} / \mathrm{SiO}_{2}$ Bragg reflector on a glass substrate. Light is incident on the structure from air at an angle of $54.3^{\circ}$. For comparison, results are also shown without the ITO (Bragg) and with a $120 \mathrm{~nm}$ ITO layer on a glass substrate in the absence of the reflector (No BR). The full loss term was included in the calculations.

FIG. 7 TE polarization. The reflection coefficient as a function of energy at an external air/interface angle of $50.0^{\circ}$. Results for the air/Bragg reflector/glass substrate (Bragg) and air/ITO(1210 nm)/Bragg reflector/glass substrate (BraggITO) structures are shown. A distinct feature associated with an SPP at the ITO/Bragg reflector 
interface is present at $1.203 \mathrm{eV}$. A nominal loss term of $0.01 \omega_{c}$ was employed in the calculations.

FIG. 8 A plot of the tangential component of the electric field intensity, $E_{\tan }^{2}$ (relative), associated with the TE SPP state clearly demonstrating the field localization at the ITO/Bragg reflector interface. The field intensity is plotted relative to that of the incident wave.

FIG. 9 The TE reflection coefficient as a function of energy for $w=10 \mathrm{~nm}, 50 \mathrm{~nm}, 100 \mathrm{~nm}$, $260 \mathrm{~nm}$ and $500 \mathrm{~nm}$ thick ITO layers on a 40 layer $\mathrm{SiO}_{2} / \mathrm{TiO}_{2}$ Bragg reflector on a glass substrate. Light is incident on the structure from air at an angle of $50.0^{\circ}$. For comparison, results are also shown without the ITO (Bragg) and with a $260 \mathrm{~nm}$ ITO layer on a $\mathrm{SiO}_{2}$ substrate in the absence of the reflector (No BR). The full loss term was included in the calculations. 
Figure 1

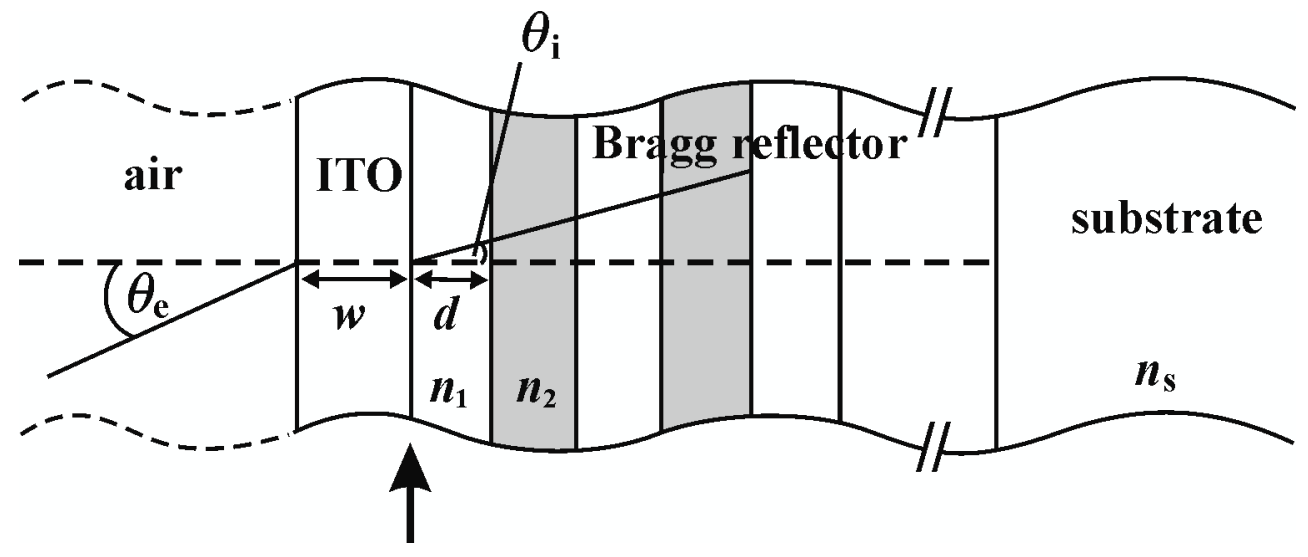


Figure 2

(a)

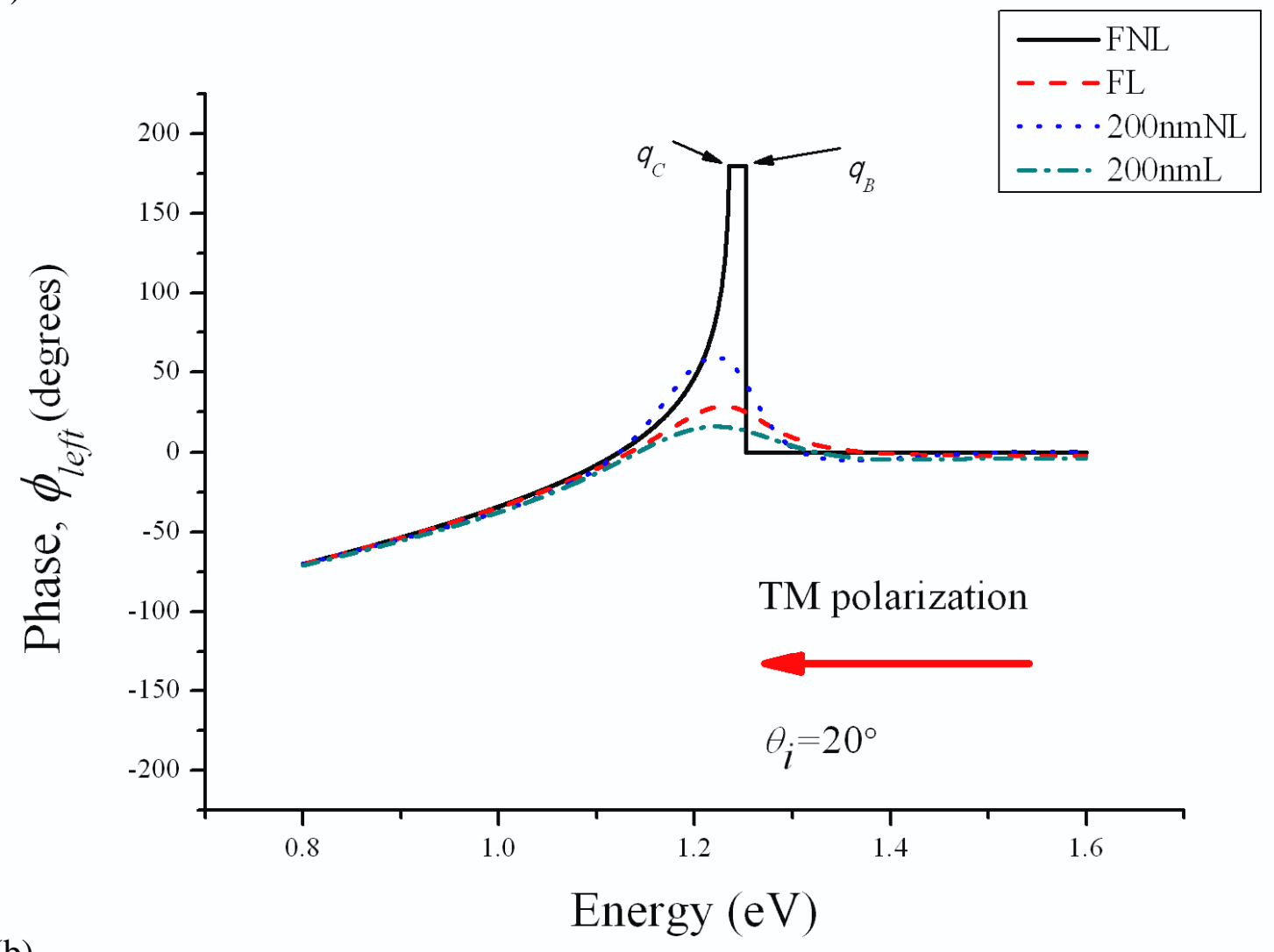

(b)

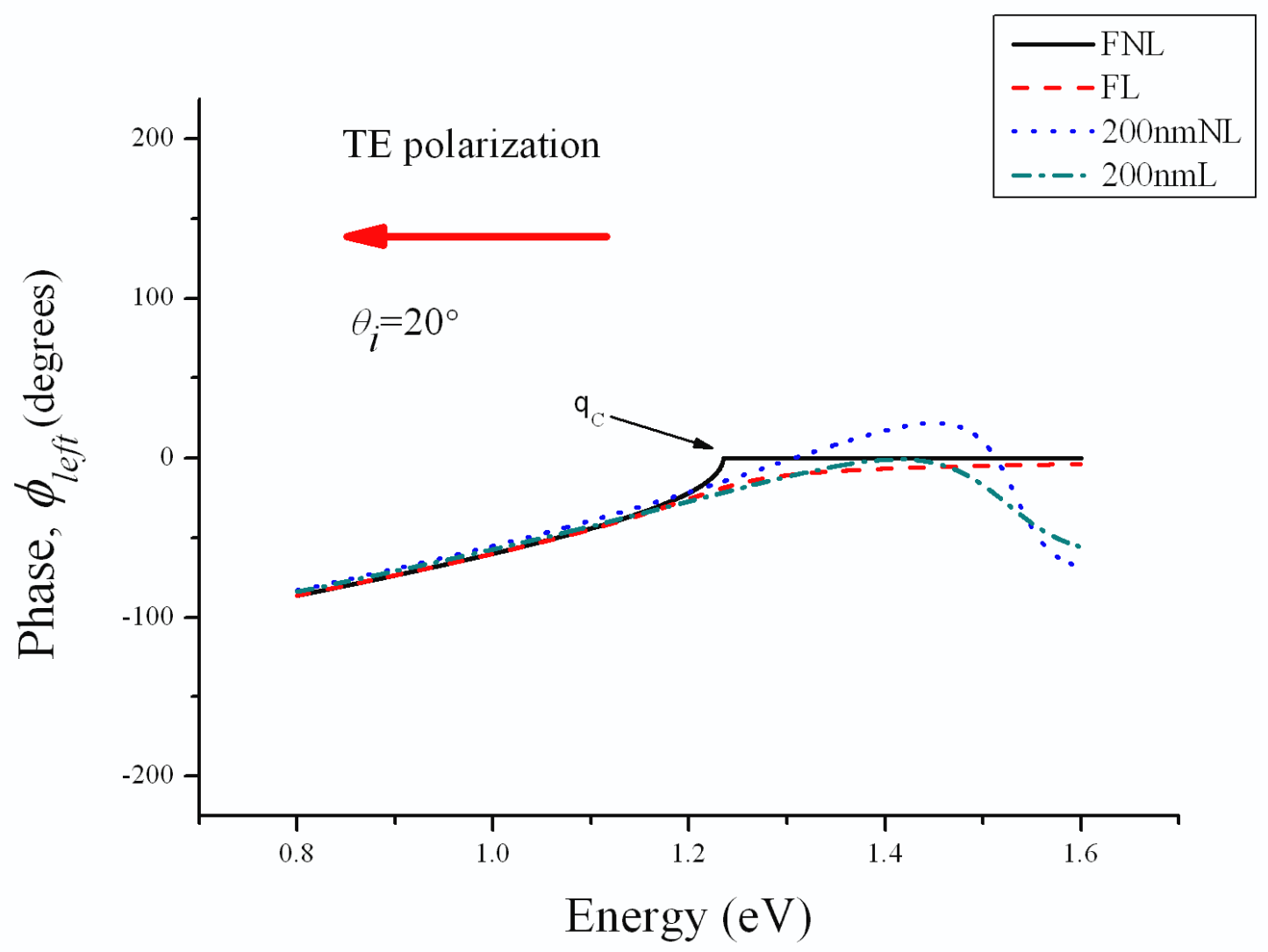


Figure 3

(a)

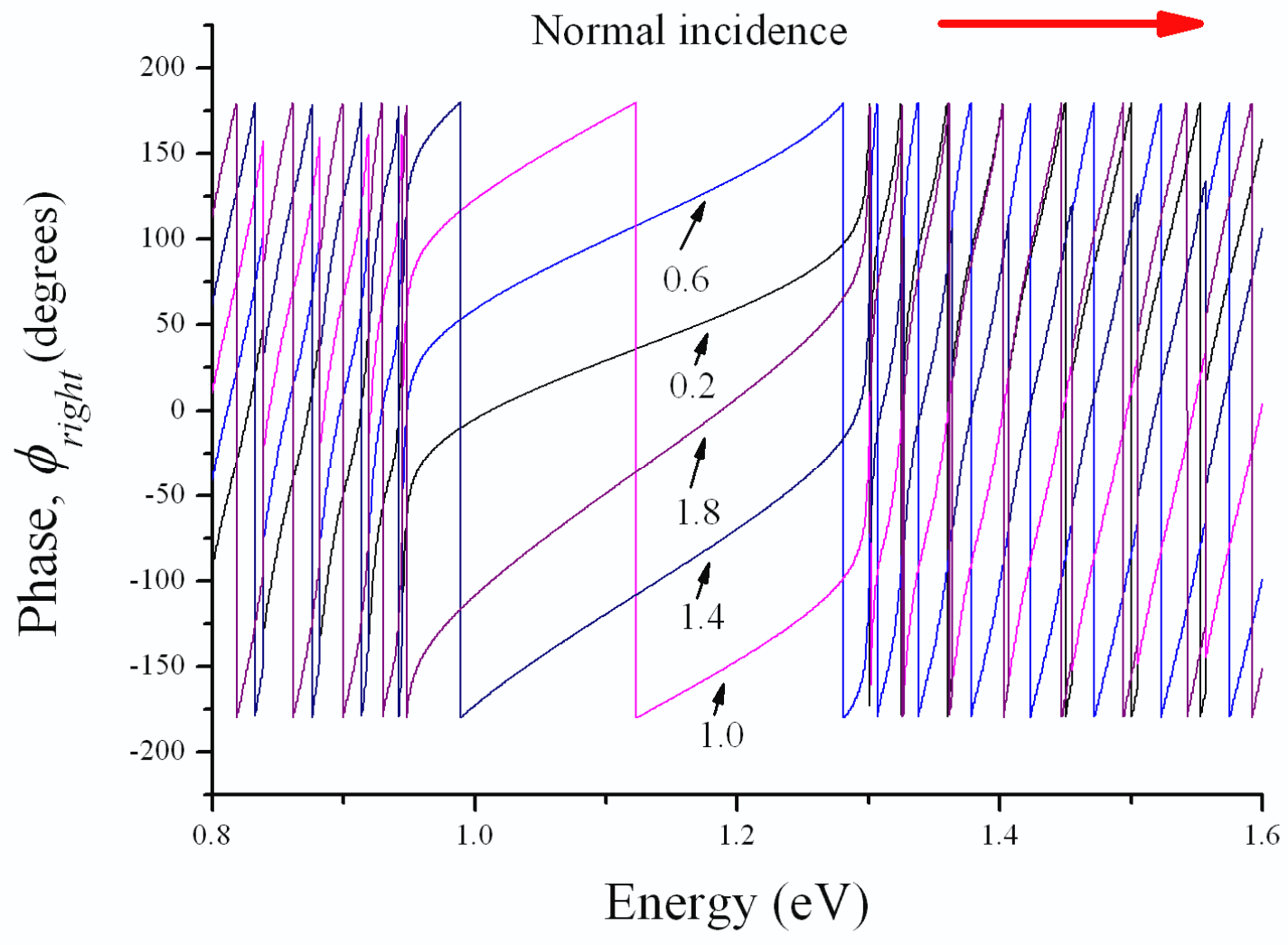

(b)

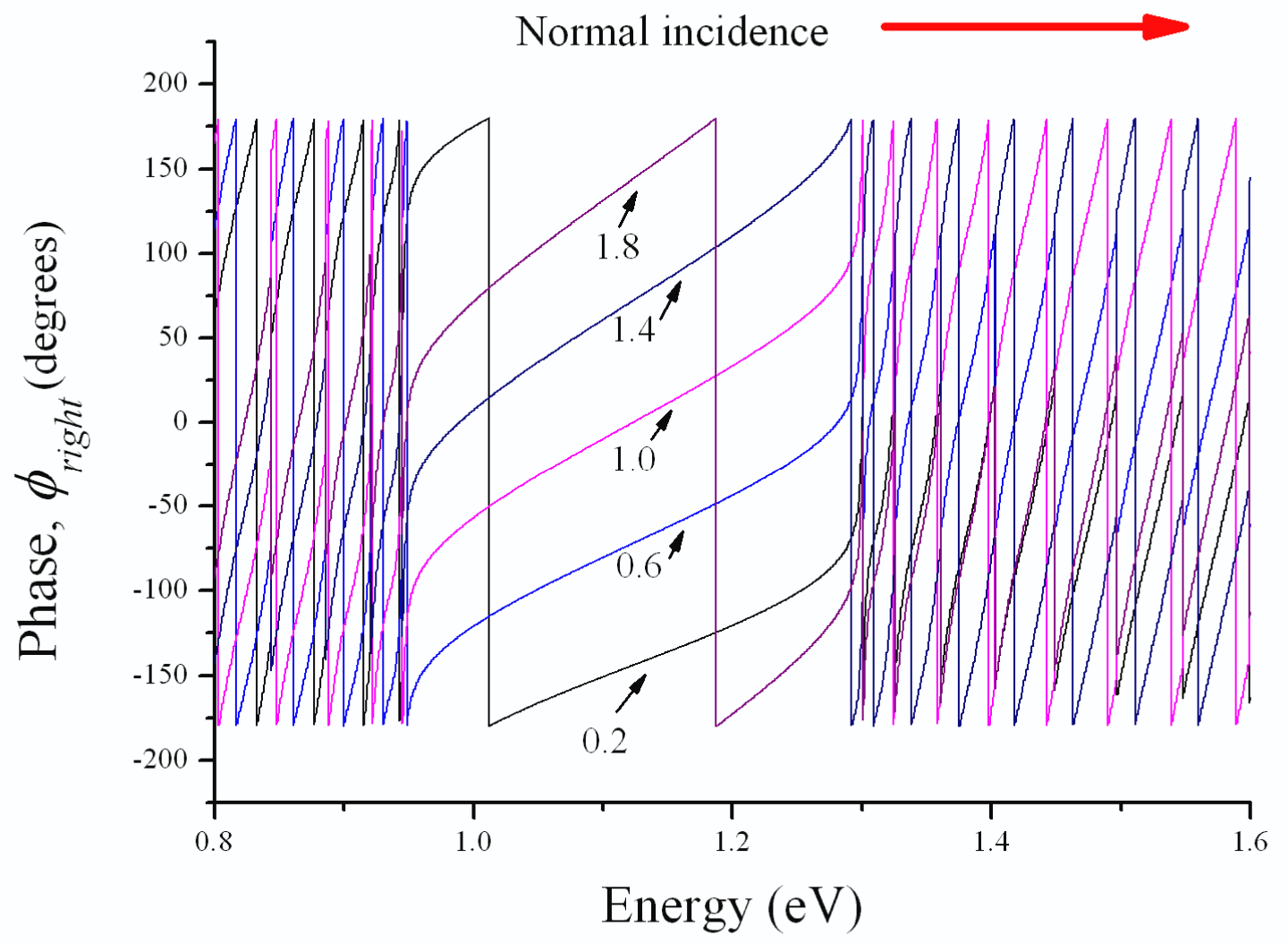


Figure 4

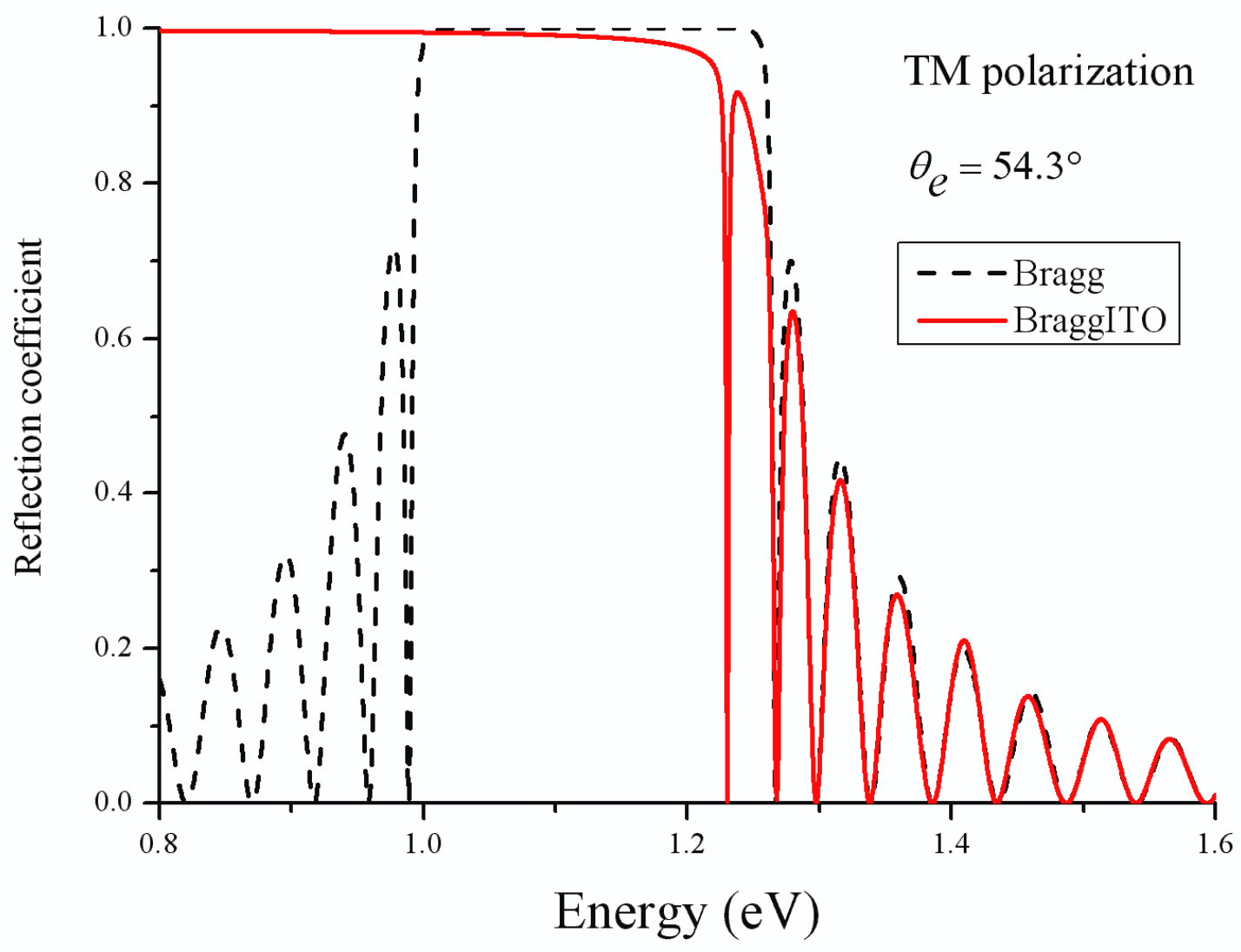


Figure 5

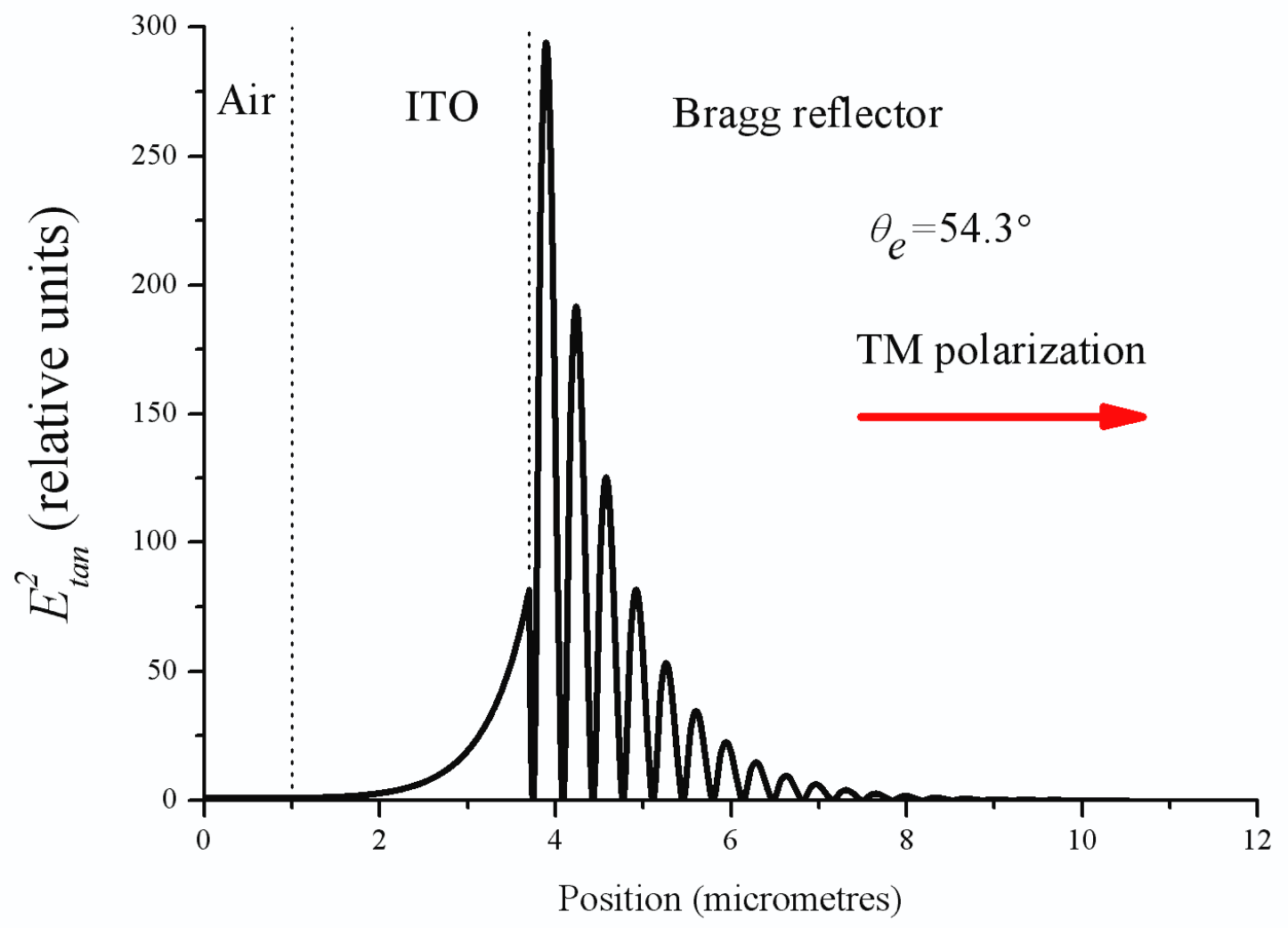


Figure 6

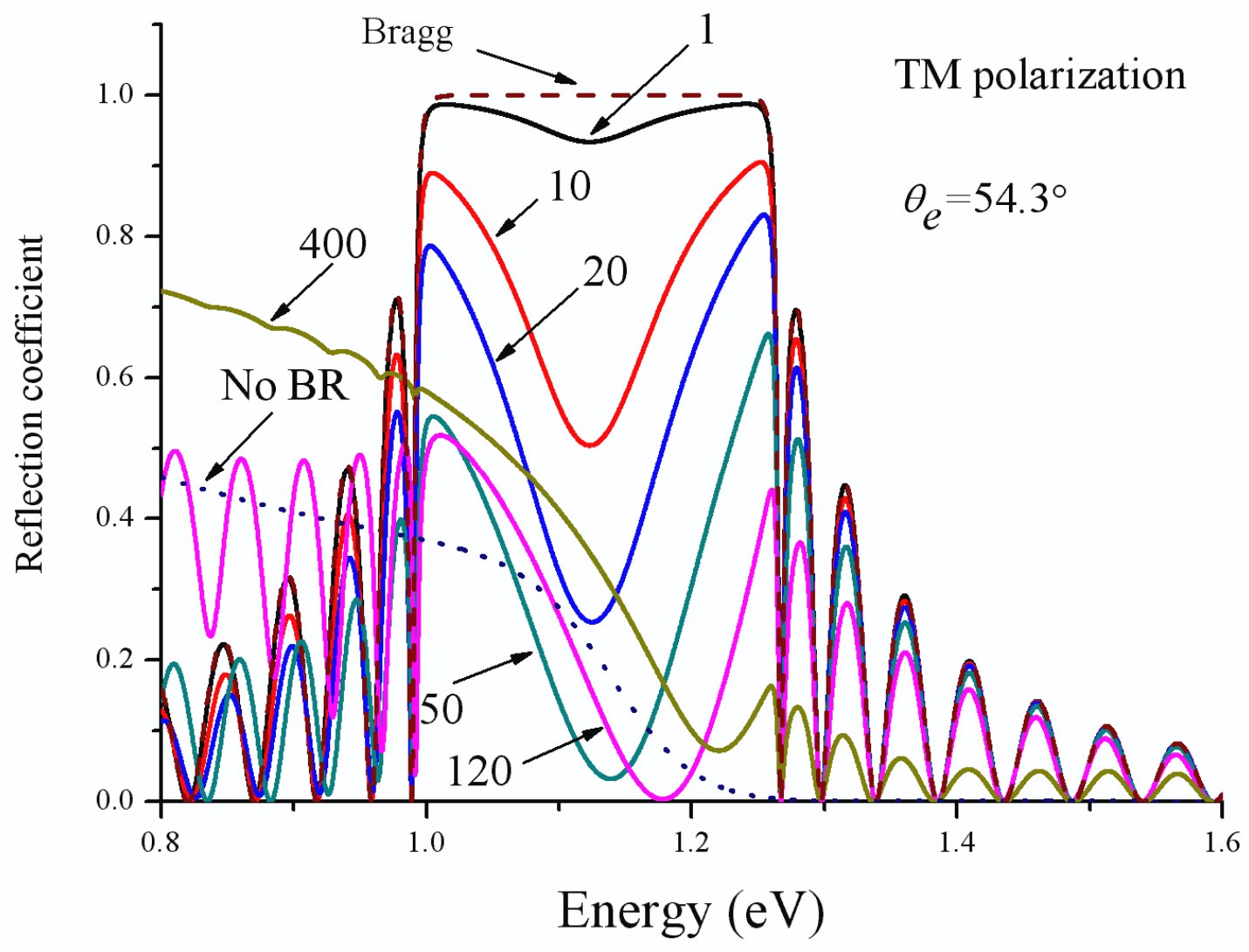


Figure 7

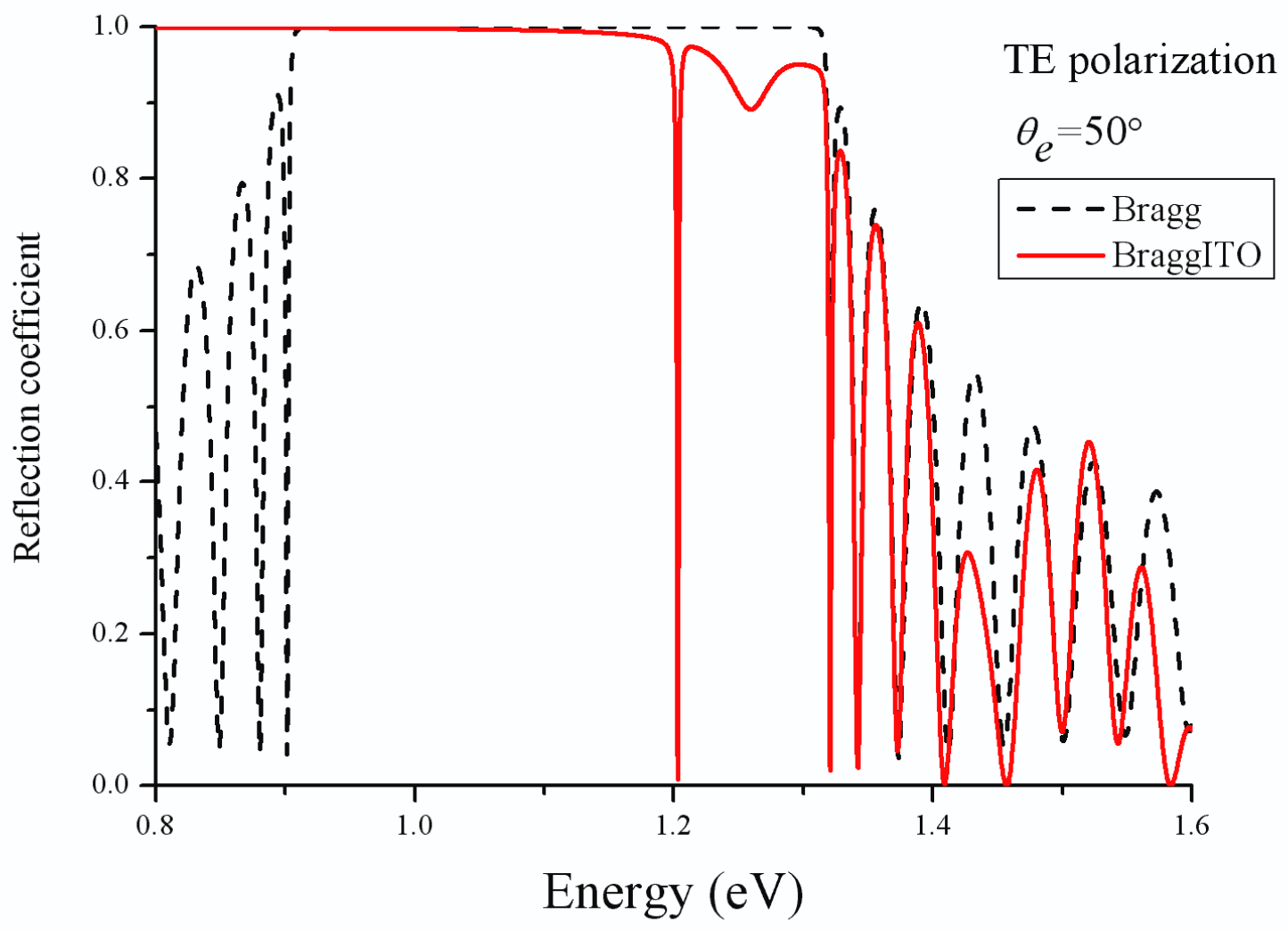


Figure 8

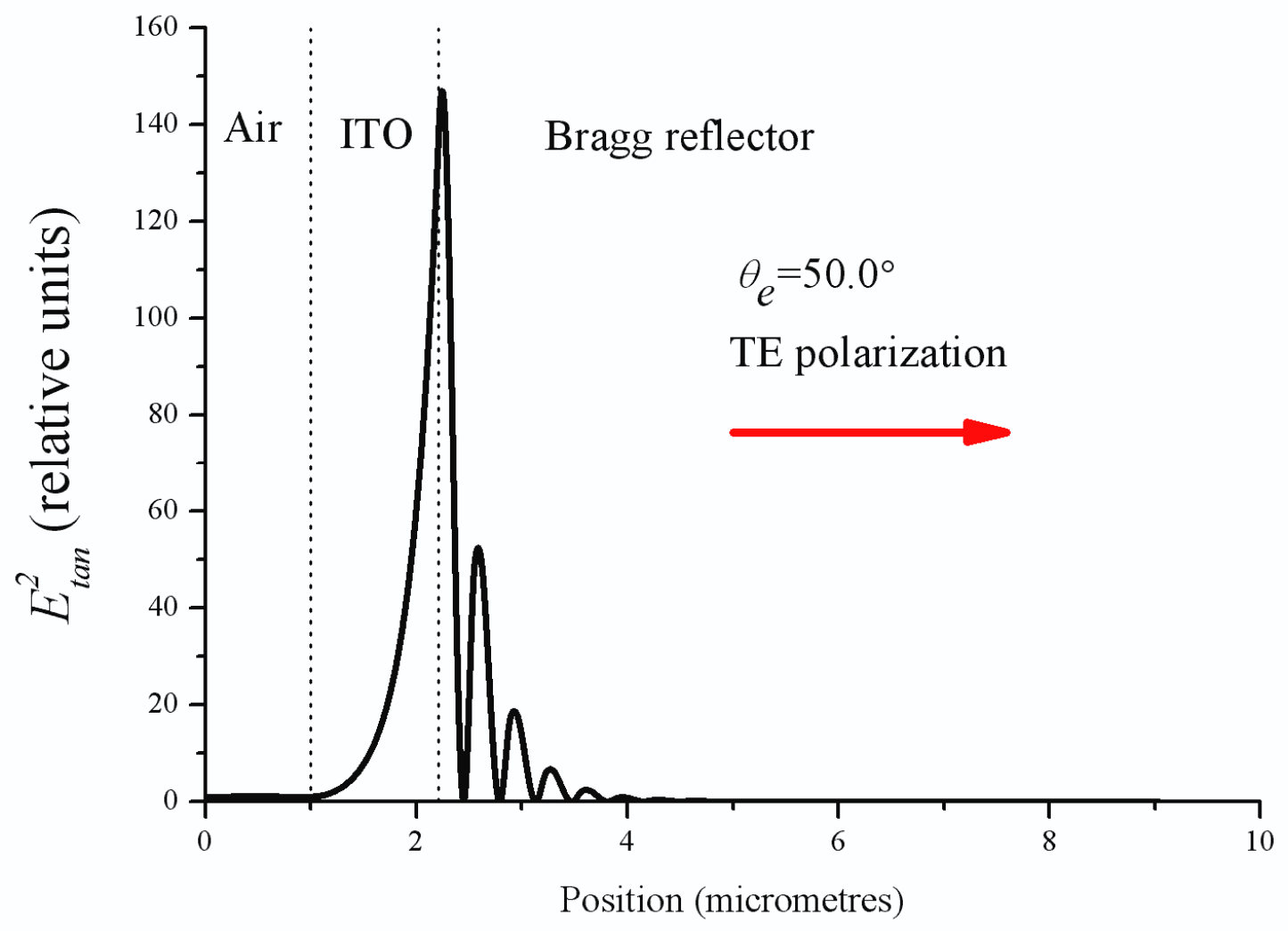


Figure 9

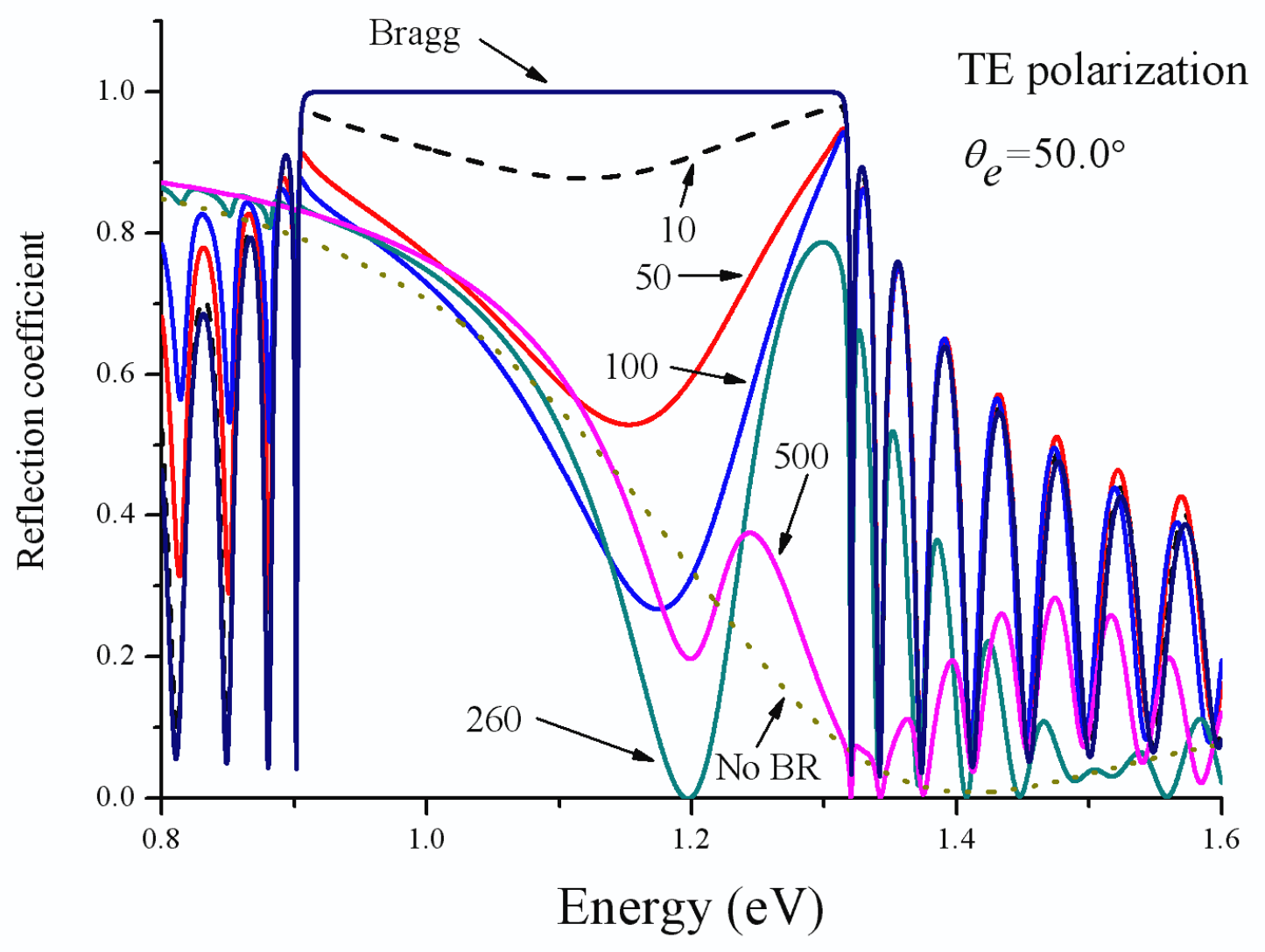

